\title{
COMPORTAMENTO E COMPOSIÇÃO DE UM GRUPO DE CALLITHRIX JACCHUS ERXLEBEN (PRIMATES, CALLITRICHIDAE) NA MATA DE DOIS IRMÃOS, RECIFE, PERNAMBUCO, BRASIL
}

\author{
Goretti Sônia da Silva ${ }^{1}$ \\ Maria Adélia O. Monteiro da Cruz ${ }^{2}$
}

\begin{abstract}
BEHAVIOR AND COMPOSITION OF A GROUP OF CALLITHRIX JACCHUS ERXLEBEN (PRIMATES, CALLITRICHIDAE) IN DOIS IRMÃOS FOREST, RECIFE, PERNAMBUCO, BRAZIL. The objective of this study was to determine the size and composition of a group of wild Callithrix jacchus Erxleben, 1777, a small neotropical primate from the North East Brazil. The study was carried out in Dois Irmãos Forest, Recife, Pernambuco, Brazil, in an area of 6 ha where this social group of marmosets had its home range. A graded transects system was cut to provide the access and moving during the observation period. To provide an individual identification for the members of the marmosets group and to achieve the objective of this study, two captures were made, with a six month interval between them in a $50 \times 50 \mathrm{~m}^{2}$ area at the nuclear range of the group's area. The animals were realeased after a maximum period of $24 \mathrm{~h}$ after traping. We made, during the whole study period, direct observations of the social group which wasn't limited only to count and identification of the marmosets, but expanded in consideration on the ecology and behavior of the Callithrix jacchus group. The results show us that the size and composition of the study group was similar to others descriptions for the Callitrichidae. The group composition suffered a $30 \%$ "turnover" in a six month interval, and the adults particulary, had a big frequency of change. One pair of infants was seen in December 1986 - in the middle of the dry season - and another infant appeared in the study group in 1987 , just after the end of the wet season. Some dietary items were fruits, leaf buds and also exudate from the trunks and branchs of some tree's species. Intergroup agonistic interactions were recorded during February 1987.
\end{abstract}

KEY WORDS. Primates, Callitrichidae, Callithrix jacchus, behavior

O sagui-do-nordeste, primata da espécie Callithrix jacchus Erxleben, 1777, pertencente à sub-ordem dos platirrinos (símios neotropicais de narinas dirigidas lateralmente e septo nasal largo), subfamília Callitrichinae, acha-se largamente distribuído por toda a região do Nordeste do Brasil, habitando praticamente todos os tipos de formações vegetacionais ali encontrados, desde dunas, restingas, mangues; passando pelas várias formas de matas (úmida, seca, serrana, etc) às zonas do Agreste e das Caatingas do Sertão. Esta plasticidade

1) Graduação no Curso de Bacharelado e Licenciatura em Ciências Biológicas pela Universidade Federal de Pernambuco.

2) Departamento de Morfologia e Fisiologia Animal, Universidade Federal Rural de Pernambuco, Av. Dom Manoel de Medeiros, 52171-900 Recife, Pernambuco, Brasil. 
se deve em grande parte a algumas características morfofisiológicas e comportamentais inerentes à espécie.

Dados recentes de campo, obtidos para outros calitriquídeos (notadamente Saguinus Hershkovitz, 1977, Cebuella Gray, 1866 e algumas outras espécies do gênero Callithrix Erxleben, 1777) mostram que os mesmos vivem em grupos flexíveis, com tamanhos que variam entre dois e 15 indivíduos, e que ocorre movimento freqüente entre os membros de grupos adjacentes. Esses movimentos afetam a composição de um determinado grupo social, tendo este fluxo de animais causas diversas. Estudos de campo posteriores, de longa duração, serão necessários para esclarecer suas razões.

Esses grupos podem conter de um a vários machos e fêmeas adultos. Quase todos os indivíduos do grupo auxiliam a mãe a carregar os filhotes, pois o nascimento de gêmeos fraternos é normalidade na subfamília Callitrichinae.

Os objetivos deste trabalho foram os de descrever o tamanho e a composição de um grupo de Callithrix jacchus em uma área que sofre perturbações devido à presença e ação humana - fortemente predatória - e suas possíveis mudanças no transcorrer do tempo, com vistas a promover subsídios para a efetivação de programas que visem a conservação da área e a preservação da espécie em questão. O parâmetro "composição de um grupo social" é investigado sob duas perspectivas; uma vez que ela é tanto um produto quanto um determinante do comportamento individual de seus membros.

\section{MATERIAL E MÉTODOS}

O local de estudo foi uma área nos limites do Horto Zoo-Botânico de Dois Irmãos, Recife, Pernambuco (Fig. 1), entre os meses de janeiro de 1986 a agosto de 1987. A área utilizada pelo grupo de estudo apresentava trechos de mata secundária, tinha cerca de 6 ha e se encontrava bastante descaracterizada no seu limite sul, em particular, devido à proximidade de habitações humanas, corte da vegetação nativa, plantio de árvores frutíferas, cultivo de hortaliças e criação de animais domésticos entre outras atividades antrópicas.

Com o objetivo de permitir um bom acesso e orientação na área de mata selecionada, foram cortadas trilhas, obdecendo um sistema de transectos em gradeado, nas direções norte-sul e leste-oeste, distanciadas 50 metros uma das outras. Nos pontos de intersecção entre as trilhas transversais foram colocadas estacas identificadas alfanumericamente de acordo com o sistema de coordenadas cartesianas, onde as trilhas horizontais receberam números e as verticais, letras do alfabeto.

Após a identificação dos limites da área domiciliar (home range), de sua respectiva área nuclear e do período de adaptação do grupo ao observador, foram realizadas capturas a intervalos de seis meses uma da outra, utilizando armadilhas automáticas de grades de metal para pequenos mamíferos modificadas (Fig. 2). Estas capturas possibilitaram o reconhecimento dos membros do grupo social, através do uso de diferentes tipos de marcação, à saber: tatuagem (Fig. 3), tricotomia circular de partes da cauda (Fig. 4) e colares de 
cordas ajustáveis, de onde pendiam pequenas placas acrílicas de várias cores, formas e identificações (Fig. 5).

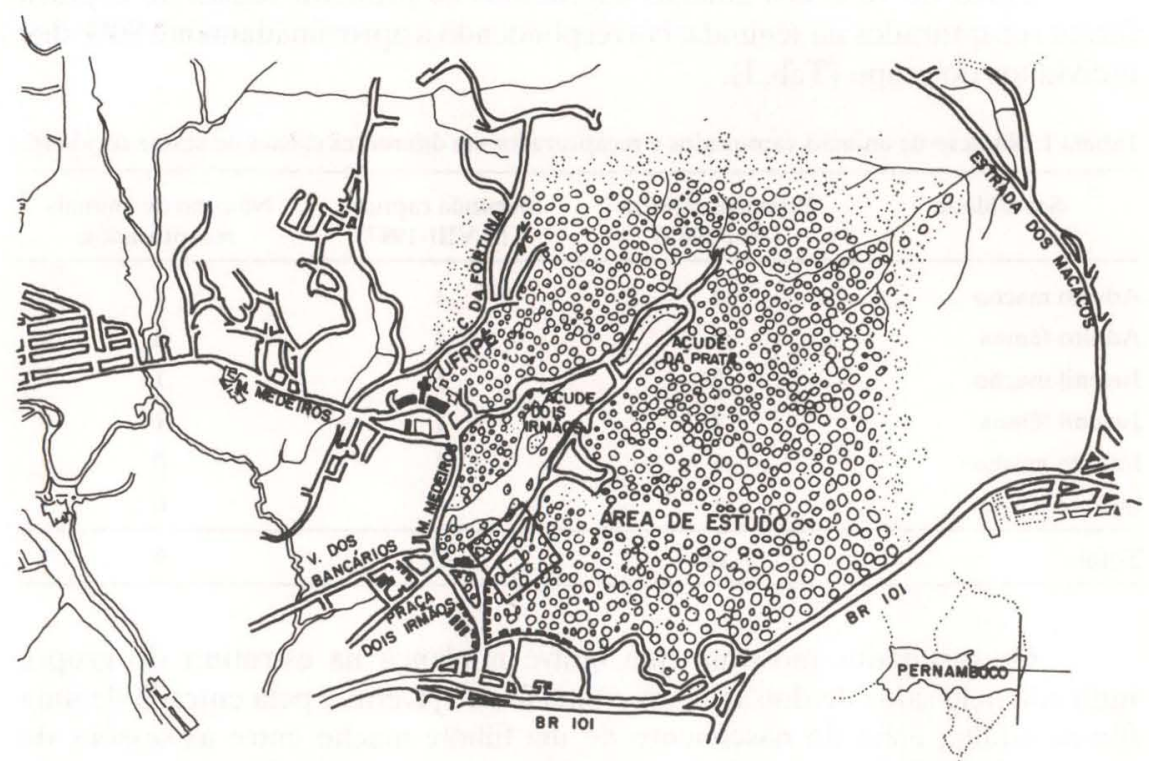

Fig. 1. Mata de Dois Irmãos, Pernambuco. Localização da área de estudo.

As contagens dos animais do grupo de estudo obtidas através das observações livres foram comparadas ao número de animais obtido nas capturas, com o objetivo de confirmar os dados de campo sobre a composição do grupo ou de qualquer mudança nos seus membros.

Dados preliminares referentes à atividades diárias dos sugüis foram registrados, incluindo: tipo e localização dos locais de pernoite, extensão e localização da área nuclear, itens da dieta alimentar, e participação e comportamento dos membros do grupo durante encontros agonísticos.

\section{RESULTADOS}

Os dados sobre o tamanho e a composição do grupo de sagüis estudado, tanto da primeira quanto da segunda captura, estão representados na tabela I. O grupo, quando capturado em janeiro de 1987, era constituído por treze indivíduos, sendo sete adultos, quatro juvenis e dois infantes; e em agosto do mesmo ano e grupo estava constituído por onze membros, sendo oito adultos, dois juvenis e um infante.

A relação entre machos e fêmeas adultas variou de um ligeiro excesso de machos na primeira captura para a igualdade entre os sexos na segunda captura. O número de juvenis capturados por grupo variou de quatro para dois e as relações entre sexos nessa classe de idade foi sempre proporcional. Compa- 
rando as duas capturas temos que entre as classes de sexo/idade se mantiveram sem alteração.

Cerca de $70 \%$ dos animais capturados na primeira sessão de captura foram recapturados na segunda, correspondendo a aproximadamente $80 \%$ dos indivíduos do grupo (Tab. I).

Tabela I. Número de animais capturados e recapturados em diferentes classes de sexo e de idade.

\begin{tabular}{lccc}
\hline Sexo/Idade & $\begin{array}{c}\text { Primeira captura } \\
(26-I-1987)\end{array}$ & $\begin{array}{c}\text { Segunda captura } \\
\text { (18-VIII-1987) }\end{array}$ & $\begin{array}{c}\text { Número de animais } \\
\text { recapturados }\end{array}$ \\
\hline Adulto macho & 4 & 4 & 4 \\
Adulto fêmea & 3 & 4 & 3 \\
Juvenil macho & 2 & 1 & 1 \\
Juvenil fêmea & 2 & 1 & 1 \\
Infante macho & 1 & 1 & 0 \\
Infante fêmea & 1 & 0 & 0 \\
\hline Total & 13 & 11 & 9 \\
\hline
\end{tabular}

Os resultados mostram que houve mudança na estrutura do grupo, indicada pela saída de dois machos e uma fêmea juvenis e pela entrada de uma fêmea adulta, além do nascimento de um filhote macho entre as sessões de captura (Tab. II). O turnover no grupo alcançou, portanto, $30 \%$ em um período de seis meses e foi mais evidente entre animais mais velhos.

Tabela II. Número de sagüis recapturados que mudaram sua classe de idade entre as duas capturas e o número de animais novos no grupo na segunda captura.

\begin{tabular}{lcccc}
\hline \multirow{2}{*}{ Sexo/Idade } & Primeira captura & \multicolumn{3}{c}{ Número de animais } \\
\cline { 2 - 3 } \cline { 5 - 5 } \cline { 5 - 5 } & Janeiro de 1986 & Recapturados & por mudança de classe & Novos \\
\hline Adulto macho & 4 & 2 & 0 \\
Adulto fêmea & 3 & 1 & 1 & 0 \\
Juvenil macho & 2 & 1 & 0 \\
Juvenil fêmea & 2 & 0 & 1 \\
Infante macho & 1 & 0 & 0 \\
Infante fêmea & 1 & 5 & 2 \\
\hline Total & 13 & &
\end{tabular}

A travessia do grupo através das pontes de vegetação e a saída do mesmo dos locais de pernoite (Fig. 6), proporcionaram momentos ideais para a contagem do número de indivíduos do grupo. Foi possível identificar dois locais utilizados pelo grupo para pernoite. Ambos eram árvores altas com cerca de 20 metros de altura, com a copa totalmente fechada devido à presença de epífitas, formando um verdadeiro emaranhado, protegendo assim o grupo de ser visua- 
lizado por seus predadores. Umas das árvores utilizadas como local de pernoite foi identificada como sendo a Bowdichia virgilioides H.B.K. (sucupira-mirim) e tinha sua copa recoberta por Philodendron imbe Schmitt (imbé).

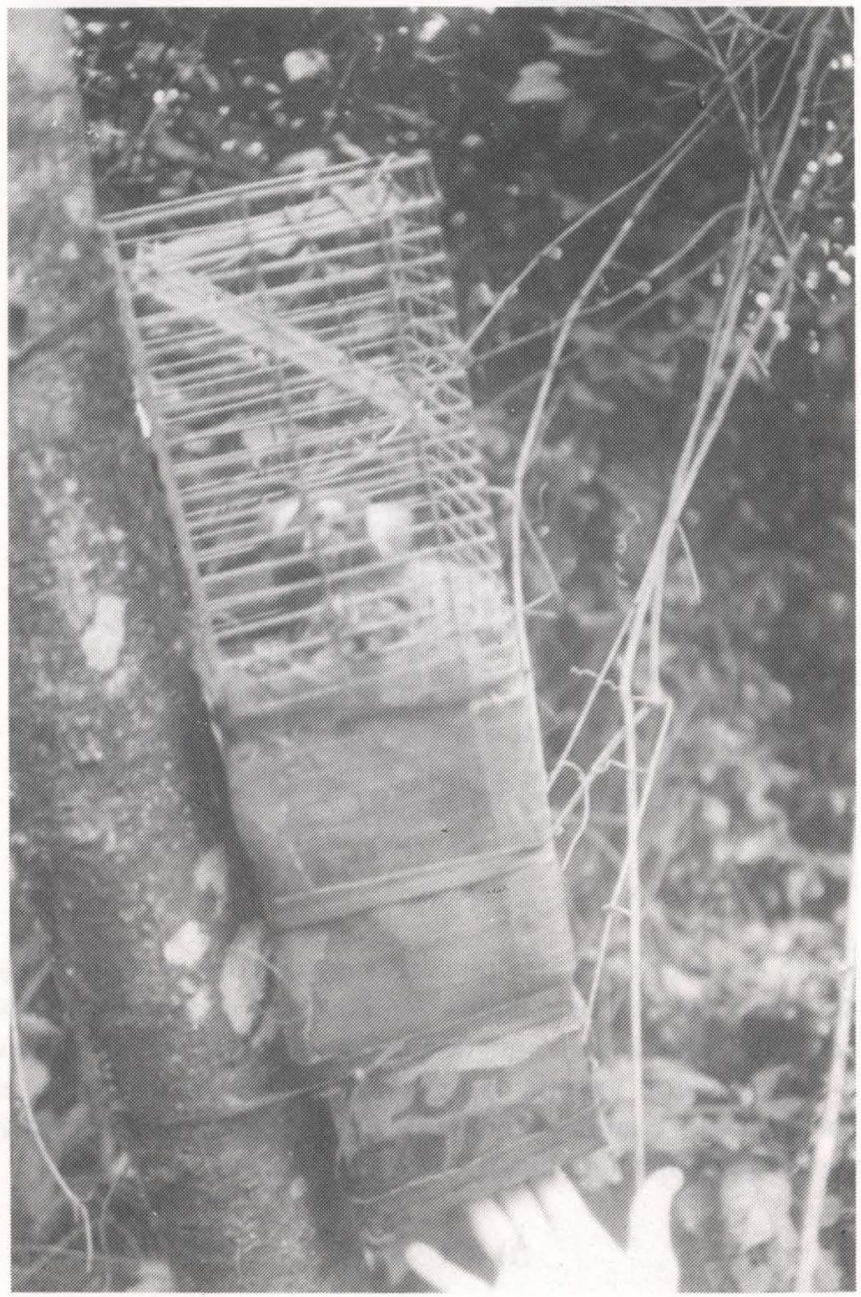

Fig. 2. Armadilha pronta para a captura.

Foi possível observar o consumo de alguns itens alimentares pelos sagüis, entre eles se destacaram: broto de folhas, polpa de frutos nativos e introduzidos, exsudatos de árvores como Tapirira guianensis Aubl. (pau-pombo) e a Anacardium occidentale Linaeus (cajueiro). A gomovoria é um comportamento característico do gênero Callithrix e os exsudatos constituíram grande parte da dieta do grupo durante o período de observação. 


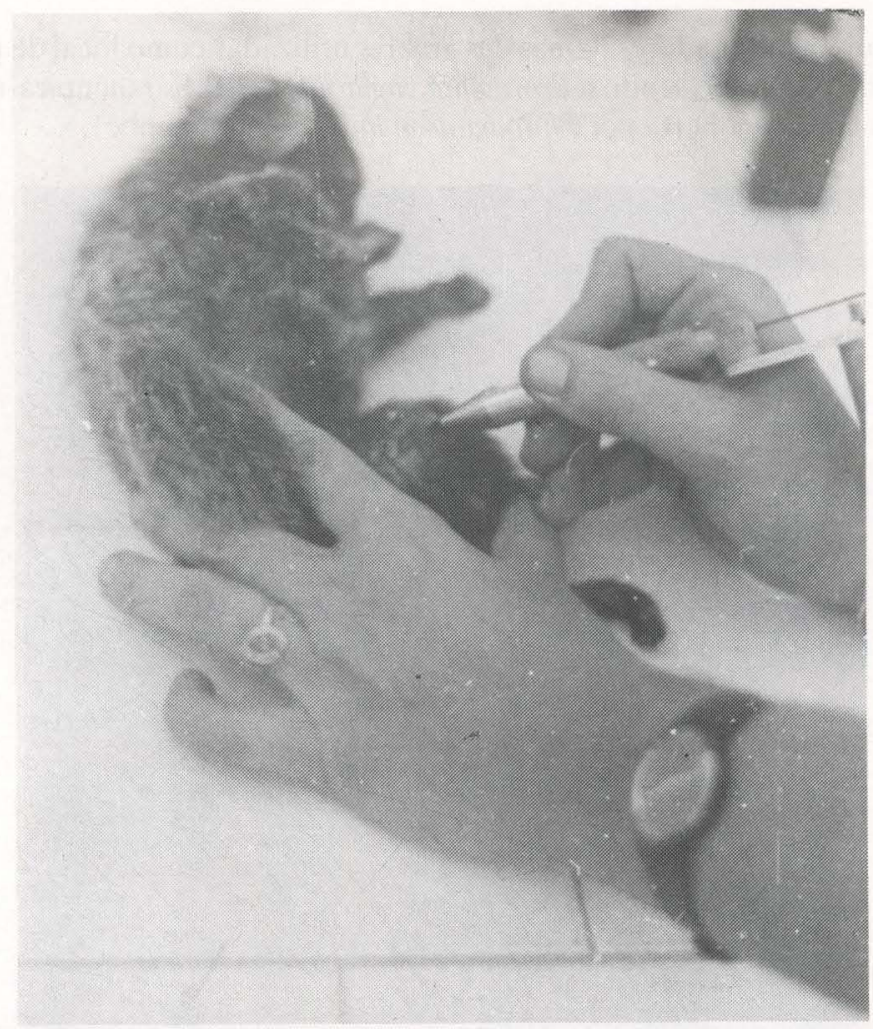

Fig. 3. Sagui sendo tatuado na parte interna da coxa.

Durante as observações foram registrados alguns encontros agonísticos entre o grupo observado e seus vizinhos. A tentativa mau sucedida de introdução antrópica de uma fêmea com um único filhote agarrado ao dorso, no dia 10-II-1987, desencadeou atitudes agonísticas por parte do grupo estudado, incluindo brigas com contatos corporais entre adultos e emissão de sinais sonoros (vocalização típica de luta).

\section{DISCUSSÃO}

Observando-se os resultados de tamanho e composição do grupo, as razões para afirmar que os animais capturados representam um grupo real de Callithrix jacchus são as seguinte: primeiro, o número de animais que foi capturado, solto e depois recapturado seis meses depois, permaneceu praticamente estável; segundo, as estimativas de tamanho do grupo obtido pelas contagens dos animais através das observações livres correspondem bem aos números obtidos em ambas sessões de captura. Terceiro, o fato de aproximadamente 70\% dos sagüis capturados na primeira captura, terem sido recapturados e corres- 


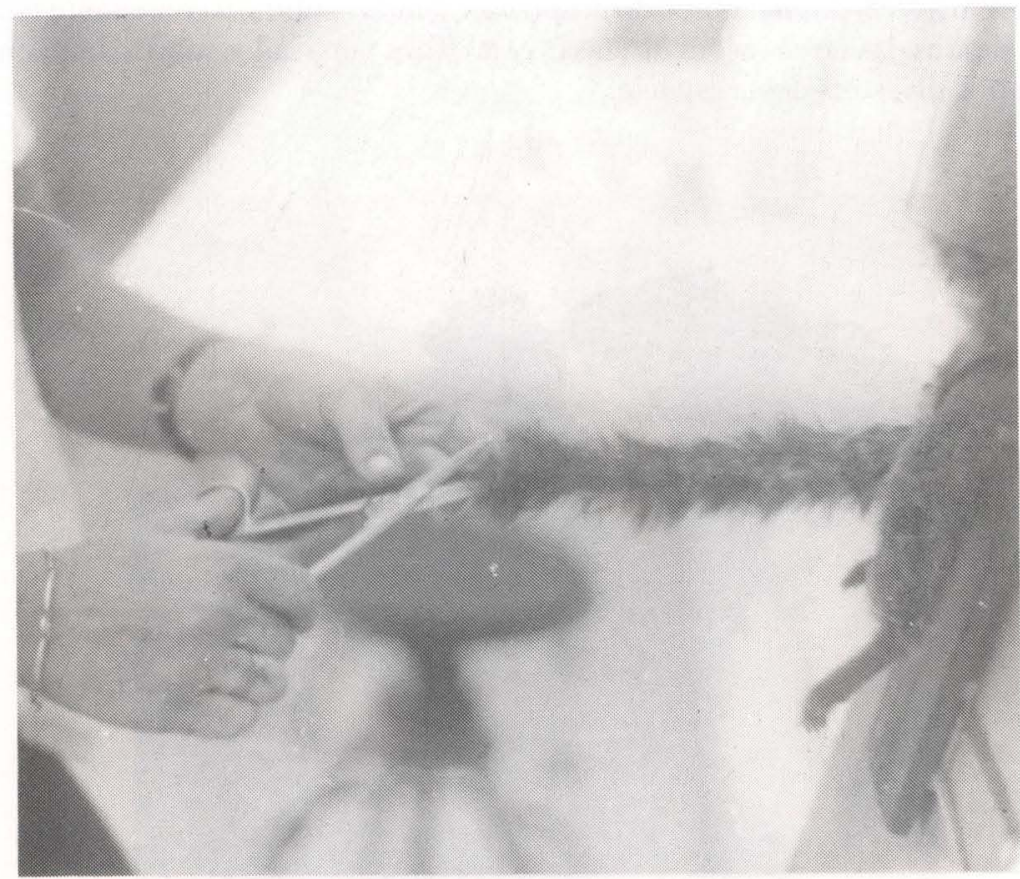

Fig. 4. Tricotomia circular de partes da cauda do sagüi.

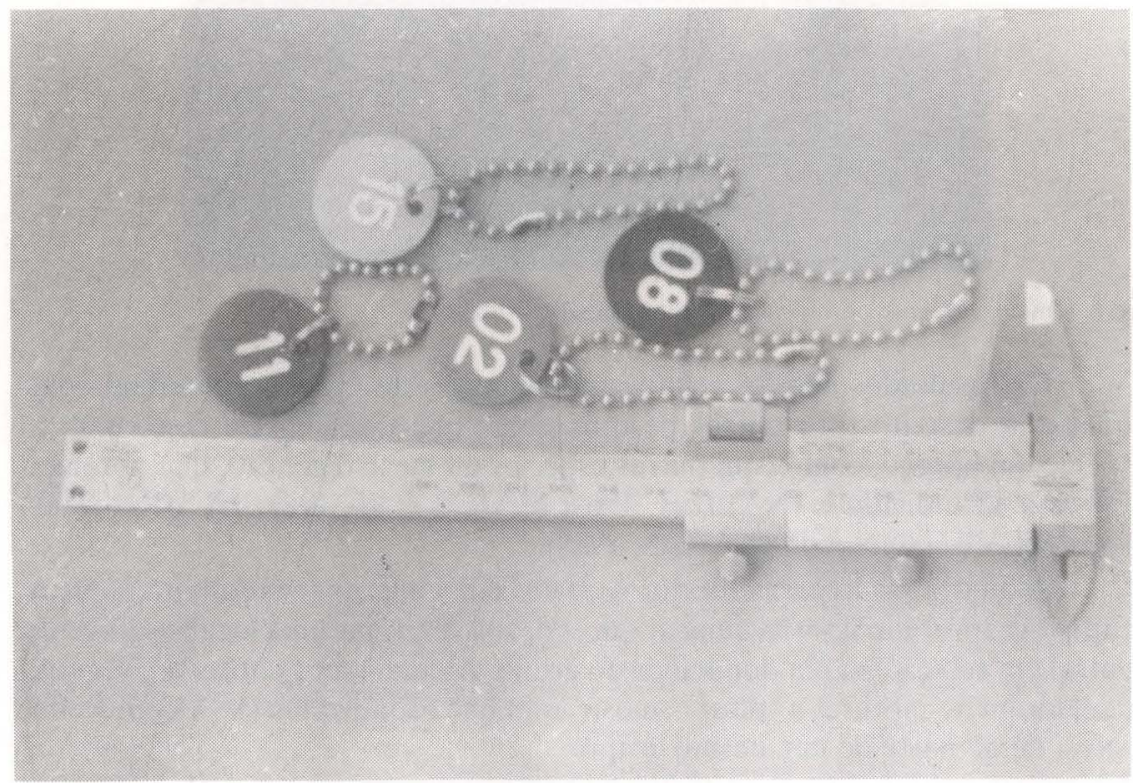

Fig. 5. Placas acrílicas de diferentes formas, cores e identificação.

Revta bras. Zool. 10 (3): 509-520, 1993 
ponderem a $80 \%$ dos membros do grupo na segunda captura, é consistente com os resultados das observações diretas e, com dados publicados sobre o tamanho de grupos silvestres dessa espécie.

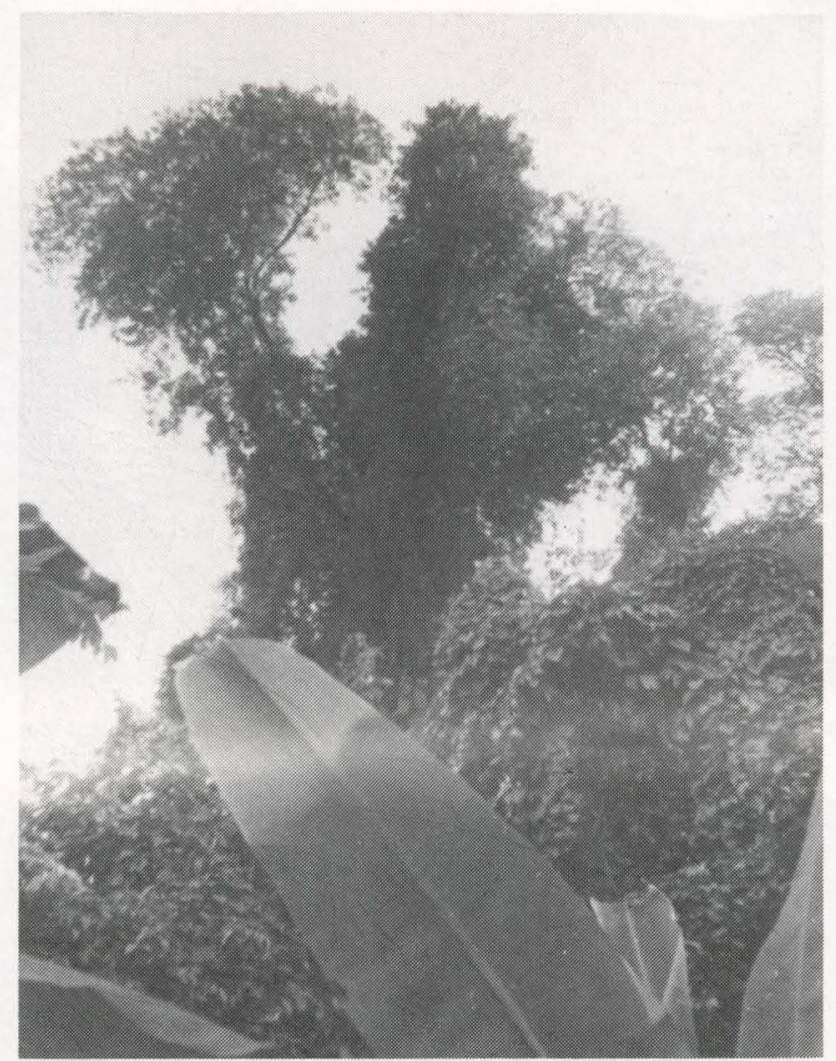

Fig. 6. Um dos locais de pernoite do grupo de sagüis estudado.

Os resultados obtidos relativos ao tamanho do grupo concordam com aqueles já descritos por outros pesquisadores que trabalharam tanto com a espécie em apreço, como com outras espécies do mesmo gênero, que abrange de três a 15 indivíduos (NAPIER, 1967; MAIER et al., 1982; HUBRECHT, 1984; STEVENSON \& RYLANDS, 1988).

A quarta razão para crer que se tratava de um grupo verdadeiro é que o grupo foi capturado na área nuclear que, por sua vez, tinha uma localização bem central em relação à área domiciliar do grupo. Além disso, as observações que se seguiram às capturas e solturas mostraram que os indivíduos se locomoviam juntos, como parte de um mesmo grupo.

Os resultados mostraram que houve mudança na estrutura do grupo. A 
amplitude do turnover para o período e a evidência da saída preferencial de adultos são dados consistentes com aqueles de SCANLON et al. $(1988,1989)$.

Esta fluidez demonstrou que apesar dos sagüis viverem em grupos que se mantêm constantes ao longo do tempo em relação ao tamanho e às proporções entre as classes de idade/sexo, houve movimento freqüente entre os indivíduos de grupos adjacentes. Os dados relativos ao número de animais em diferentes classes de idade/sexo, que não sofreu alteração com o fluxo de animais, são inconsistentes com aqueles registrados por SCANLON (1988).

Alguns autores concordam que os sagüis possuem um sistema social do tipo monogâmico, com apenas um par reprodutor, onde o número de filhotes por nascimento varia de um a três animais - o mais comum é a reprodutora dar a luz a gêmeos fraternos - e a gestação se prolonga por cerca de 140 a 150 dias (NAPIER \& NAPIER, 1967; BOX, 1977; ALONSO, 1984; RYLANDS, 1982).

$\mathrm{O}$ fato de terem sido capturados dois filhotes na primeira captura e um filhote na segunda, leva a crer que apesar do grupo possuir mais de um par de adultos, provavelmente, apenas uma fêmea estava reproduzindo por vez.

Estudando um outro calitriquídeo, o Saguinus fuscicollis Hershkovitz, 1977, TERBORGH \& WILSON (1985) registraram a emigração da fêmea reprodutora do grupo, levando com ela os filhotes. No estudo atual, a provável fêmea reprodutora (fêmea adulta, placa azul) emigrou do grupo, contudo seus filhotes permaneceram.

Hipóteses recentes sobre a fluidez dos grupos de Callithrix indicam que as fêmeas têm um período curto como reprodutora no grupo e que, em conseqüência, seria mais vantajoso para elas mudarem mais freqüentemente do que os machos. Os resultados desse trabalho não fornecem evidências para tal hipótese. Estudos que impliquem no acompanhamento de um número maior de grupos serão necessários para esclarecer esta questão.

Os registros de comportamento alimentar obtido nesse estudo são similares aqueles publicados sobre o gênero Callithrix. Nos gêneros Callithrix e Cebuella a disposição dos dentes na arcada dentária possibilita a perfuração e escavação do lenho de árvores e cipós para a obtenção de exsudatos (NAPIER \& NAPIER, 1967; COIMBRA-FILHO \& MITTERMEIER, 1976, 1977; ROSENBERGER, 1978; GARBER, 1980; COIMBRA-FILHO et al., 1981; THORINGTON \& ANDERSON, 1984). A gomivoria constitui a maior parte das observações sobre itens alimentares. As espécies vegetais Tapirira guianensis e Anacardium occidentale foram as mais utilizadas neste sentido e foram visitadas e utilizadas durante todo o ano, o que está de acordo com os dados obtidos por MAIER et al. (1982) e MELLO (1985).

Os resultados obtidos nesse estudo levam a concordar com aqueles autores que consideram a atividade roedora como uma característica essencial para esta espécie, por possibilitar a aquisição de um recurso constante e disponível ao longo de todo o ano, tornando os sagüis independentes do período de safra (FONSECA et all., 1984; SANTEE \& FARIA, 1985).

Os encontros agonísticos, nos limites do território, observados entre o 
grupo estudado e seus vizinhos, foram pouco freqüentes, no entanto os resultados mostraram que foram semelhantes em relação às ameaças e displays agressivos obtidos em outros estudos com a mesma espécie de primata. Estas ameaças também envolveram movimentos rápidos e posturas típicas do agressor na direção de indivíduos de outros grupos e emissão de sinais sonoros repetidos como foi descrito por EPPLE (1968) para Callithrix jacchus em cativeiro e por RYLANDS (1979) para Callithrix humeralifer intermedius Hershkovitz, 1977 no ambiente natural.

Apesar de habitar um ambiente perturbado devido à ação antrópica, o grupo estudado de Callithrix jacchus não apresentou nenhuma característica de seu repertório comportamental que se mostrasse afetado por este motivo. Este fato fortalece a efetivação do conceito de grande plasticidade atribuída a esta espécie.

\section{CONCLUSÕES}

Dos resultados obtidos conclui-se que: - o grupo capturado representou um grupo real de Callithrix jacchus selvagens; - o número de animais por grupo foi similar aqueles descritos para a espécie em trabalhos anteriores; - foi registrado um número máximo de dois infantes por grupo; - o turnover dos membros do grupo alcançou $30 \%$ em um período de tempo de aproximadamente seis meses; - os animais jovens tenderam a permanecer no grupo;- houve freqüente movimento entre os indivíduos dos grupos estudados e esta fluidez deveu-se, principalmente, à emigração dos animais adultos; - a proporção de animais nas diferentes classes de idade/sexo não se alterou com o fluxo de animais; - a gomivoria no grupo de Callithrix jacchus estudado mostrou-se ser uma atividade imprescindível para sua sobrevivência, sendo as espécies vegetais Tapirira guianensis (pau-pombo) e Anacardium occidentale (cajueiro) utilizadas intensivamente durante todo $o$ ano de estudo.

\section{REFEÊNCIAS BIBLIOGRÁFICAS}

ALONSO, C. 1984. Observações de campo sobre o cuidado à prole e o desenvolvimento dos filhotes de Callithrix jacchus, p.67-78. In: M.T. DE MELLO (ed.). A primatologia no Brasil. I. Brasília, Sociedade Brasileira de Primatologia.

BOX, H. 1977. Quantitative data on carrying of young captive monkeys (Callithrix jacchus) by other members of their family group. Primates 18 (2): 475-484.

COIMBRA-FILHO, A.F. \& R.A. MITTERMEIER. 1976. Exudate-eating and three-gouging in marmosets, Nature, London, 262: 630.

. 1978. Tree-gouging, exudate-eating and the "short-tusked" conditions in Callithrix and Cebuella, p.105-115. In: D.G. KLEIMAN (ed.). Washington D.C., Smithsonian Instituition Press.

COIMBRA-FILHO, A.F.; R.A. MITTERMEIER \& I.D. CONSTABLE. 1981. 
Callithrix flaviceps (Thomas, 1903) recorded from Minas Gerais, Brasil (Callitrichidae, Primates). Rev. Bras. Biol. 41: 141-147.

COIMBRA-FILHO, A.F.; N. DA C. ROCHA \& A. PISSINATTI. 1980. Morfofisiologia do cêco e sua correlação com o tipo odontológico em Callitrichidae (Platyrrhini, Primates). Rev. Bras. Biol. 40: 177-185.

EPPLE, G. 1968. Comparative studies of vocalization in marmoset monkeys (Hapalidae). Folia Primatologica 8: 1-40.

FARIA, D.S. 1984. Aspectos Gerais do Comportamento de Callithrix jacchus penicillata em mata ciliar do cerrado, p.56-65. In: M.T. DE MELLO (ed.). Brasilia, Sociedade Brasileira de Primatologia.

FONSECA, G.A.B.; T. LACHER; C. ALVES JR. \& B. MAGALHÃES CASTRO. 1980. Some ecological aspects of free-living black tufted-ear marmosets (Callithrix jacchus penicillata). Antropologia Contemporânea 3: 197.

FONSECA, G.A.B. 1981. Utilização de exsudatos de espécies arbóreas do cerrado pelo mico-estrela (Callithrix jacchus penicillata). Resumos do VIII Congresso Brasileiro de Zoologia 8: 171-172.

GARBER, P.A. 1980. Locomotor behavior and feeding ecology of the Panamanian tamarin (Saguinus oedipus geoffroyi, Callitrichidae, Primates). Int. J. Primatol. 1: 185-201.

HUBRECHT, R.C. 1984. Field observations"on group size and composition of the common marmoset (Callithrix jacchus jacchus) at Tapacurá, Brazil. Primates 25 (1): 13-21.

MAIER, W.; C. ALONSO \& A. LANGGUTH. 1982. Field observations of Callithrix jacchus. L. Z. Saugetierkunde 47: 334-346.

MELLO, M.T. DE. 1985. Atividade roedora de Callithrix penicillata (sagui mico estrela). In: A.B. RYLANDS (ed.). A Primatologia do Brasil II. Belo Horizonte, Sociedade Brasiliera de Primatologia.

NAPIER, J.R. \& P.H. NAPIER. 1967. A handbook of living Primates. New York, Academic Press.

RIZZINI, C.T. \& A.F. COIMBRA-FILHO. 1981. Lesões produzidas pelo sagüi Callithrix penicillata (E. Geoffrey, 1812) em árvores do cerrado (Callitrichidae, Primates). Rev. Bras. Biol. 41 (3): 579-583.

ROSENBERGER, A.L. 1978. Loss of incisor enamel in marmosets. Journ. Mammology 59: 207-208.

RYLANDS, A.B. 1977. Observações preliminares sobre o sagüi Callithrix humeralifer intermedius (Hershkovitz, 1977) em Dardanelos, Rio Aripuanã, Mato Grosso. Acta Amazônica 9 (3): 589-602.

1982. The behaviour and ecology of three species of marmosets and tamarins (Callitrichidae, Primates) in Brasil. Dissertação de Doutorado, não publicada, University of Cambridge, Cambridge U.K.

SANTEE, D. \& D.S. FARIA. 1985. Padrōes de comportamento utilizados pelos sagüis (Callithrix jacchus penicillata) na retirada de exsudato. Psicologia 11 (1): 65-74. 
SCANLON, C.E.; N.R. CHALMERS \& M.A.O. MONTEIRO DA CRUZ. 1988. Changes in size, composition and reproductive condition of wild marmosets group (Callithrix jacchus) in Northeast Brazil. Primates 29 (3): 295-305.

- 1989. Home range use and the exploration of gum in the marmoset Callithrix jacchus jacchus. Intern. Journ. Primatology 10 (2): 123-136.

STEVENSON, M.F.\& A.B. RYLANDS. 1988. The marmosets, genus Callithrix. In: R. MITTERMEIER; A.B. RYLANDS; A.F. COIMBRA-FILHO \& G.A.B. FONSECA (eds). Ecology and Behaviour of Neotropical Primates. 2. Washington, World Wildlife Fundation.

TERBORGH, J. \& A. WILSON. 1985. On the mating system of the cooperatively breeding saddle-backed tamarin (Saguinus fuscicollis). Behav. Ecolo. Sociobiol. 16: 293-299.

THORINGTON, R.W. \& S. ANDERSON. 1984. p. 187-217. In: S. ANDERSON \& I.K. JONES JR. (eds). Primates. John Wiley \& Sons Inc.

WINTER, M. 1978. Allagrooming relationships in capture cammon marmosets (C. jacchus jacchus), p. 161-179. In: H. ROTHE; H.I. WALTERS \& J.P. HEARN (ed.). Biology and behaviours of marmosets. Göttingen, Eigenverlog Rothz.

Recebido em 31.XII.1991; aceito em 25.XI.1993. 